\title{
IMPROVEMENT OF THE CRUISE PERFORMANCES OF A WING BY MEANS OF AERODYNAMIC OPTIMIZATION. VALIDATION WITH A FAR-FIELD METHOD
}

\section{J. Jiménez-Varona and J. Ponsin Roca}

INTA, Aerodynamics Department Carretera Ajalvir Km.4,5 Torrejón de Ardoz, Madrid, Spain

Under a contract with AIRBUS MILITARY (AI-M), an exercise to analyze the potential of optimization techniques to improve the wing performances at cruise conditions has been carried out by using an in-house design code. The original wing was provided by AI-M and several constraints were posed for the redesign. To maximize the aerodynamic efficiency at cruise, optimizations were performed using the design techniques developed internally at INTA under a research program (Programa de Termofluidodinámica). The code is a gradient-based optimization code, which uses classical finite differences approach for gradient computations. Several techniques for search direction computation are implemented for unconstrained and constrained problems. Techniques for geometry modifications are based on different approaches which include perturbation functions for the thickness and/or mean line distributions and others by Bézier curves fitting of certain degree. It is very important to afford a real design which involves several constraints that reduce significantly the feasible design space. And the assessment of the code is needed in order to check the capabilities and the possible drawbacks. Lessons learnt will help in the development of future enhancements. In addition, the validation of the results was done using also the well-known $T A U$ flow solver and a far-field drag method in order to determine accurately the improvement in terms of drag counts.

\section{INTRODUCTION}

The CIRCE code is a wing design code, capable to handle constraints, either of geometrical or aerodynamic nature. This code has been used for the improvement of cruise aerodynamic performances of a military transport aircraft type wing.

This is an Open Access article distributed under the terms of the Creative Commons Attribution License 4.0, which permits unrestricted use, distribution, and reproduction in any medium, provided the original work is properly cited. 
The idea was to investigate the possibilities of this type of methods to improve a wing design. Then, there were several constraints for the optimization which reduced significantly the feasible design space. The wing loading was fixed, also the wing span. There was a room for varying the leading edge sweep angle or for twist distribution. The wing taper ratio was bounded. It was checked in parametrical studies that the chosen taper ratio was close to the optimum. And there was very little improvement in varying the original one. A minimum thickness-to-chord ratio was imposed by structural and volume reasons.

The goal was to improve the aerodynamic efficiency at cruise condition. Multipoint design case was also initially afforded. The target was improving the efficiency both at cruise and at low altitude. But the limitation of the period of study avoided a systematic multipoint design.

In order to validate the optimization, a fine analysis of both the original and the resultant wing was done using also another flow solver as the $T A U$ code, available at INTA under an agreement of cooperation with DLR. An in-house tool, called DragON, was used for the drag computation. It uses a far-field method for the calculation and breakdown of the drag force.

The results confirmed an improvement of the original wing, reducing the drag at cruise condition. It is important to remark that the induced drag was not significantly reduced, as the wing span was fixed, and the wing surface was also fixed. The original wing loading was close to the elliptical loading; the Oswald factor was close to the optimum. The final one was very similar. As the cruise condition was at subsonic flow, there is no wave drag. Then, only the viscous drag component was reduced via modifying the wing sections geometry.

\section{CIRCE CODE: DESCRIPTION}

The $C I R C E$ code is a multipoint, multiobjective gradient-based optimization code, capable to handle constraints, either of geometrical or aerodynamic nature. It is focused on airfoil/wings geometries; additionally, it has been used for blended wing body - generated by sections — design or also for simple wing-body geometries.

This code has been developed within an internal research programme (Programa de Termofluidodinámica) and was applied in its former version in VELA project of the V European Framework Programme (2002-2005) for the optimization of a blended-wing-body [1]. The optimizer uses gradient-based iterative methods for determining the minimum of a certain cost function. There is need in one step to calculate both a search direction and the proper step size for the reduction of the cost function value. Several methods for determining the search direction are implemented. For unconstrained problems, steepest descent, conjugate gradients, and the Broyden family of quasi-Newton meth- 
ods are implemented. For active or violated constraints, a modified feasible directions methods developed by Vanderplaats $[2,3]$ is implemented, along with a Newton-SQP (sequential quadratic programming) method for the case with active constraints. Step size is computed with polynomial interpolation. For the wing surface modification, there is the option to make plan form variations by changing the wing leading edge sweep angle, or the law of the chords, or twist distribution. The wing sections can be modified using either perturbation functions based on thickness and mean line variations, or using Bézier parameterizations of the wing sections.

A simple method for mesh generation and remeshing - also for mesh deformation - is used, and the flow solver is capable to solve the Euler/RANS (Reynolds-averaged Navier-Stokes) equations.

A brief description of several features of this design code is given below.

\subsection{Cost Functions}

The cost functions used are typically global aerodynamic coefficients: $C_{L}, C_{D}$, $C_{M}, C_{D i}$, and also $\int_{\Sigma}\left(C_{p}(\vec{x})-C_{p_{\text {obj }}}(\vec{x})\right)^{2} d S$ being $C_{p_{\text {obj }}}(\vec{x})$ a target pressure coefficient at wing surface.

Multipoint cases are formulated using the cost function $f(\vec{x})=\sum_{i=1}^{N} \omega_{i} f_{i}(\vec{x})$ being $\sum_{i=1}^{N} \omega_{i}=1$ and $f_{i}(\vec{x})$ the cost function at each design point.

Multiobjective problems can be formulated using a pseudoobjective function as $f(\vec{x})=\sum_{i=1}^{N} v_{i} f_{i}(\vec{x}), f_{i}(\vec{x})$ being several cost functions. For example, one function can be the lift coefficient for low speed conditions and another function can be the drag coefficient for cruise. The proper values for the weighting parameters are part of the solution making use of the Pareto frontier method. But for a practical design, this is not affordable for a problem which depends on a very large number of design variables. Then, they can be chosen by the user depending on the type of objective and the balance of the different cost functions in the design procedure.

\subsection{Design Variables}

The flow field and then the global coefficients are the functions of the wing shape and of the flow conditions. These flow conditions are the altitude, the Mach number, angle of attack, Reynolds number, etc. 
The Reynolds number depends on the Mach number, the altitude, and the mean aerodynamic chord. Then, these parameters can be used as design variables; also, the angle of attack can be used as design variable. In fact, in the authors' code, only angle of attack or chords are actually used as design variables. The altitude has an influence in multipoint cases and the velocity is usually not modified. All the variables that define the wing shape are also design variables. The wing shape is parameterized. Typically, the wing sections are formed by only two or three airfoils. And these airfoils can be Bézier curves of certain degree that depend on the control points' position. So, there is a discrete set of variables that define the optimization problem. In fact, this number can be very large: each airfoil shape can depend on 25-35 design variables, for example. Then, it is possible to have a set of $75-100$ variables for a complete wing design. This involves a very large number of computations.

For a practical design, it is convenient to choose the smallest number of design variables possible to afford the problem. But it must be taken into account that a reduced number of design variables reduces also the feasible design space and makes the final solution more dependent on the initial wing geometry.

The wing plan form is modified by variations of several values as the leading edge sweep angle, the chord law, or twist law. Linear variation is utilized for the sweep angle and piecewise linear variations are used for the chord or twist laws.

The variables used for the wing sections geometry are introduced by using several perturbation or parameterization functions that will be described below.

\subsubsection{Perturbation functions}

An airfoil section can be defined by a thickness and a mean line distributions. Perturbation functions of the initial distributions are then introduced.

One set of basic functions for the deformation of the thickness distribution is implemented in the following form:

$$
f_{t i}(x)=4 x^{p\left(x_{m i}\right)}\left(1-x^{p\left(x_{m i}\right)}\right)
$$

with

$$
p\left(x_{m i}\right)=\frac{\log (1 / 2)}{\log \left(x_{m i}\right)}, \quad 0 \leq x_{m i} \leq 1 .
$$

The variable $x$ defines the dimensionless coordinate and is bounded as: 0 $\leq x \leq 1$. The value $x_{m i}$ is the percentage of the chord where the maximum of $f_{t i}(x)$ is located. The parameter $x_{m i}$ may be expressed as

$$
x_{m i}=\frac{i}{N_{1}+1}, \quad i=1, \ldots, N_{1},
$$

$N_{1}$ being the number of the selected functions. 
For the mean line, the following functions are used:

$$
f_{c j}(x)=4(1-x)^{p\left(x_{m j}\right)}\left(1-(1-x)^{p\left(x_{m j}\right)}\right)
$$

with

$$
p\left(x_{m j}\right)=\frac{\log (1 / 2)}{\log \left(1-x_{m j}\right)}, \quad 0 \leq x_{m j} \leq \frac{1}{2},
$$

and

$$
f_{c j}(x)=4 x^{p\left(x_{m j}\right)}\left(1-x^{p\left(x_{m j}\right)}\right)
$$

with

$$
p\left(x_{m j}\right)=\frac{\log (1 / 2)}{\log \left(x_{m j}\right)}, \quad \frac{1}{2} \leq x_{m j} \leq 1 .
$$

An expression for $x_{m j}$ similar to that of the thickness functions may be used, $N_{2}$ being the number of selected functions.

These basic functions are used as perturbation functions to the initial solution. The deformation of the wing section should be:

$$
\Delta f(\vec{x})=\sum_{i=1}^{N_{1}} a_{i} f_{t i}(\vec{x})+\sum_{j=1}^{N_{2}} b_{j} f_{c j}(\vec{x}),
$$

the coefficients $a_{i}$ and $b_{j}$ being the design variables that scale the value of these functions.

The plots of these functions for a number of 5 design variables for the thickness functions and 4 design variables for the mean line functions are shown in Fig. 1.

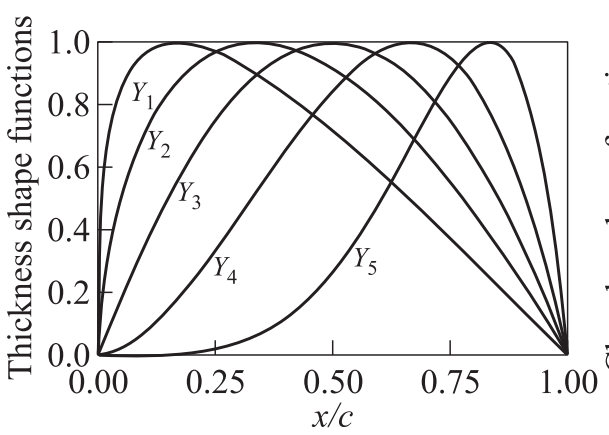

(a)

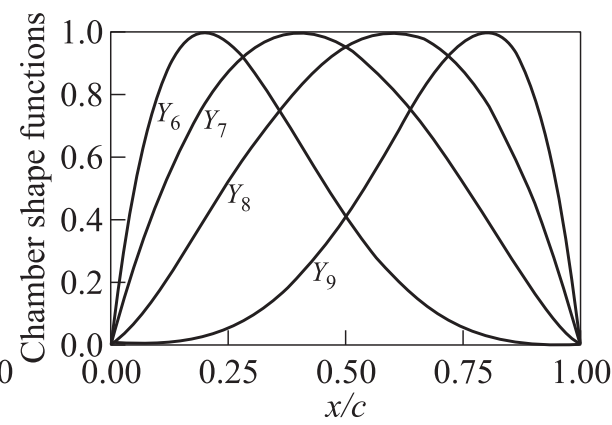

(b)

Figure 1 Thickness and mean line shape perturbation functions 


\subsubsection{Bézier curves fitting}

For the second type of modifications, a method for fitting the initial wing sections to a set of Bézier polynomials has been developed and implemented into the code.

One function is used for each side of the airfoil; at the leading edge, the continuity of the first and the second derivatives is imposed. Then, the wing sections coordinates may be approximated by

$$
Y_{u, l}(s)=\sum_{i=0}^{N_{b}} Y_{u, l}^{B}\left(\begin{array}{c}
N_{b} \\
i
\end{array}\right) s^{i}(1-s)^{N_{b}-i}
$$

where $Y$ are the fitted coordinates; $N_{b}$ is the number of control points; and $s$ ranges between 0 and 1 . The subscripts $u$ and $l$ denote upper and lower surfaces, respectively.

By solving two least square problems, the control points $Y_{u, l}^{B}$ are computed. This can be done by numerical optimization or by directly solving the following system of algebraic equations:

$$
\left[B_{i, j}\right]\left\{Y^{B}\right\}=\left\{B_{Y_{i}}\right\} \quad i, j=1, \ldots, N_{b}-1,
$$

where

$$
\begin{aligned}
& A_{i, j}=\sum_{k=1}^{N_{p}}\left[\left(\begin{array}{c}
N_{b} \\
i
\end{array}\right)\left(\begin{array}{c}
N_{b} \\
j
\end{array}\right) s_{k}^{i+j}\left(1-s_{k}\right)^{2 N_{b}-(i+j)}\right] ; \\
& B_{Y_{i}}=\sum_{k=1}^{N_{p}}\left[\left(\begin{array}{c}
N_{b} \\
i
\end{array}\right) s_{k}^{i}\left(1-s_{k}\right)^{N_{b}-i}\left(y_{0}\left(s_{k}\right)-y_{0}\left(s_{1}\right)\left(1-s_{k}\right)^{N_{b}}-y_{0}\left(s_{N_{p}}\right) s_{k}^{N_{b}}\right)\right] ;
\end{aligned}
$$

$N_{p}$ is the number of points that define the wing section; and $y_{0}$ are the coordinates of the initial wing section.

The control points $Y_{u, l}^{B}$ define the design variables.

Figure 2 shows a wing section - solid curve - and the control points of a quartic curve - in each side - used to fit it. Changing the control points then modifies these Bézier curves $Y_{u, l}^{B}$.

The use of 25 control points is usually needed to have a good initial fit of most of standard airfoils - like NACA (National Advisory Committee for Aeronautics), NASA (National Aeronautics and Space Administration) series that shape a wing section of a transport type aircraft. Then, 21 design variables per wing sections are needed for the design, when using this technique; there is a link in the leading edge, and the trailing edge points are fixed in the authors' codes.

Another possibility is to use the function for the whole section, not each side of the section. There is no need to impose any condition at the leading edge. The same fitting method is also used to obtain the control points $Y^{B}$. 


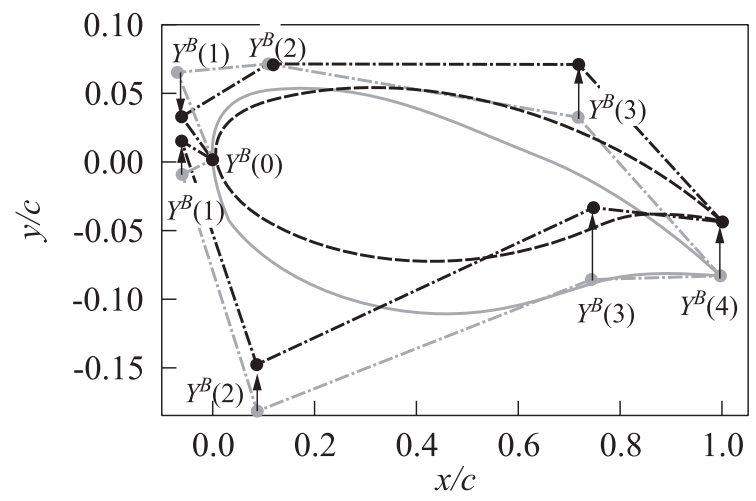

Figure 2 Wing section and control points of two quartic Bézier curves. The changes in control points lead to a new wing section

\subsubsection{Nonuniform rational $B$-spline approximation}

In this case, the purpose is to approximate the initial wing section by cubic Bézier splines curves. In this case, the number of control points is ranging between a chosen value and a maximum possible value depending on the number of points that define the initial wing section. If $N_{p}$ is the number of points (odd number) defining the initial section, then the initial wing section will be interpolated by a cubic $B$-spline in $L+1$ data that depend also on the maximum chosen number of design variables. Usually, this value may be of order 20 given the wing section by a maximum of 180 data points. These $L+1$ data are the points of the wing section: $x_{i}=b_{3 i}, i=0, \ldots, L$.

The purpose is to obtain the control points $d_{i}$ of a polygon that interpolates a cubic $B$-spline curve in these $L+1$ data.

Then, a tridiagonal system is built up such that

$$
\left[\begin{array}{ccccc}
1 & & & & \\
& \alpha_{1} \beta_{1} \gamma_{1} & & & \\
& & \ddots & & \\
& & & \alpha_{L-1} \beta_{L-1} \gamma_{L-1} & \\
& & & & 1
\end{array}\right]\left[\begin{array}{c}
d_{0} \\
d_{1} \\
\vdots \\
d_{L-1} \\
1
\end{array}\right]=\left[\begin{array}{c}
r_{0} \\
r_{1} \\
\vdots \\
r_{L-1} \\
r_{L}
\end{array}\right]
$$

where

$$
\begin{aligned}
r_{0} & =x_{1} ; \\
r_{i} & =\left(\Delta_{i-1}+\Delta_{i}\right) x_{i}, \quad i=1, \ldots, L-1 ; \\
r_{L} & =b_{3 L-1} .
\end{aligned}
$$


The values $\Delta_{i}$ are calculated as

$$
\Delta_{i}=s \_\operatorname{arc}(i+1)-s, \_\operatorname{arc}(i),
$$

this last value being the arc length at every wing section point, starting at the inner trailing edge.

The parameters $\alpha_{i}, \beta_{i}$, and $\gamma_{i}$ (with $\Delta_{-1}=\Delta_{L}=0$ ) are defined by

$$
\begin{aligned}
\alpha_{i} & =\frac{\Delta_{i}^{2}}{\Delta_{i-2}+\Delta_{i-1}+\Delta_{i}} ; \\
\beta_{i} & =\frac{\Delta_{i}\left(\Delta_{i-2}+\Delta_{i-1}\right)}{\Delta_{i-2}+\Delta_{i-1}+\Delta_{i}}+\frac{\Delta_{i-1}\left(\Delta_{i}+\Delta_{i+1}\right)}{\Delta_{i-1}+\Delta_{i}+\Delta_{i+1}} ; \\
\gamma_{i} & =\frac{\Delta_{i-1}^{2}}{\Delta_{i-1}+\Delta_{i}+\Delta_{i+1}} .
\end{aligned}
$$

With the system previously solved, the control points of this Bézier polygon define a $B$-spline curve that approximates the initial wing section and interpolates in the $L+1$ data points. The design variables are the coordinates of this control polygon.

Usually, an approximation with a $B$-spline curve of enough $L+1$ control points gives an estimated error below the manufacturing tolerance $\left(2 \cdot 10^{-4}\right)$.

In Fig. 3, it is shown (by solid curve 1 ) the initial wing section and the approximation with a $B$-spline of $L+1=19$ points. The total number of design variables is 32 . The trailing edge is fixed, and there are two coordinates per control point. The cubic $B$-spline interpolated in these points (dashed curve 4) is compared to the initial section. There is a visible difference in the inner side,

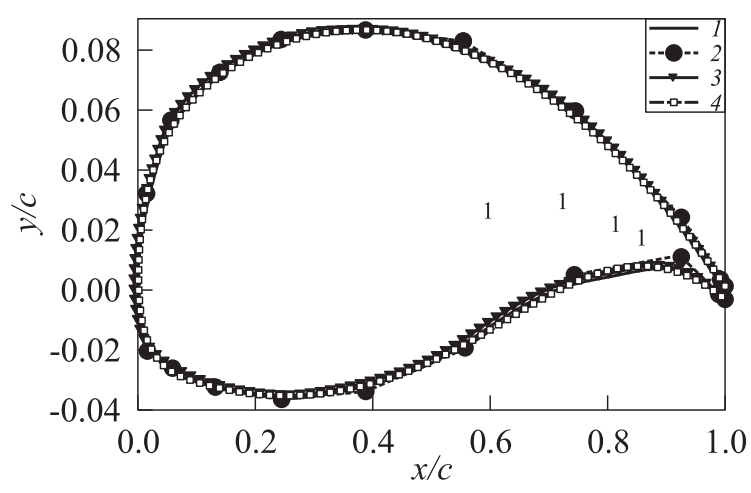

Figure 3 Wing section and approximation with a cubic $B$-spline curve. Control polygon is also shown: 1 - initial wing section; $2-$ control points; $3-B$-spline approximations; and $4-$ cubic $B$-spline interpolation 
Table 1 List of geometric and aerodynamic constraints implemented into the $C I R C E$ code

\begin{tabular}{|c|c|}
\hline Geometric constraints & Aerodynamic constraints \\
\hline 1. Minimum/maximum wing area & 1. Minimum/maximum lift coefficient \\
\hline 2. Minimum/maximum chord length & $C_{L}$ \\
\hline 3. Minimum/maximum twist & 2. Minimum/maximum pitching mo- \\
\hline 4. Minimum/maximum $(t / c)_{m}$ & inimum/maximum bendino mo- \\
\hline 5. Minimum/maximum $x / c$ position of & ment coefficient \\
\hline 6 Minimum/maximum wing section & 4. Minimum $C_{p}$ \\
\hline $\begin{array}{l}\text { 6. Minimum/maximum wing section } \\
\text { area }\end{array}$ & 5. Maximum $\operatorname{Grad}\left(C_{p}\right)$ \\
\hline $\begin{array}{l}\text { 7. Minimum/maximum area in a wing } \\
\text { section }\end{array}$ & $\begin{array}{l}\text { 6. Minimum/maximum } C_{l} \text { in a wing } \\
\text { section }\end{array}$ \\
\hline $\begin{array}{l}\text { 8. Minimum/maximum leading edge } \\
\text { radius in a wing section }\end{array}$ & $\begin{array}{l}\text { 7. Minimum/maximum } C_{m} \text { in a wing } \\
\text { section }\end{array}$ \\
\hline $\begin{array}{l}\text { 9. Minimum/maximum trailing angle } \\
\text { in a wing section }\end{array}$ & $\begin{array}{l}\text { 8. Minimum/maximum } C_{p} \text { in a wing } \\
\text { section }\end{array}$ \\
\hline $\begin{array}{l}\text { 10. Minimum/maximum mean line an- } \\
\text { gle in a wing section }\end{array}$ & \\
\hline
\end{tabular}

close to the trailing edge area. But the total error of approximation is $2.8 \cdot 10^{-4}$. For a closer approximation, the number of data $L$ should be increased. But it is enough for many purposes to start with this approximation and then to perform the optimization by changing the control polygon.

\subsection{Constraints}

There are many geometrical and aerodynamic constraints implemented into the code. Some of the constraints may be global like the wing lift coefficient. Other constraints are local like the lift coefficient in one section of the wing. For numerical calculations, it is very important to normalize these constraints, as different constraints involve different orders of magnitude.

In Table 1, there is a list of many of the constraints implemented.

\subsection{Flow Solver and Meshing Strategy}

The main features of the flow solver which is used by the CIRCE design code can be summarized in Table 2 . 
Table 2 Features of $C I R C E$ code

\begin{tabular}{ll}
\hline \multicolumn{1}{c}{ Features } & \multicolumn{1}{c}{ Description } \\
\hline Discretization technique & Finite volume, cell centered \\
\hline Grids & Single grid, structured C-H type \\
\hline Space/time discretization & - Central differencing \\
& - Jameson's type second and fourth difference dis- \\
& sipation operator \\
& - Explicit multistage Runge-Kutta scheme \\
\hline Convergence acceleration & - Local time stepping \\
& - Residual averaging \\
& - Enthalpy damping \\
& - Full multigrid $(V$-cycle, $W$-cycle $)$ \\
\hline Special features & - Time accurate version \\
& - Modified Baldwin-Lomax turbulence model \\
& - Wall function \\
\hline
\end{tabular}

Single structured grids are generated using an algebraic mesh generation procedure. Within the design procedure, the grids are regenerated using the new wing geometry. It is also possible to read in $\mathrm{C}-\mathrm{H}$ single structured grids and then the original grid is deformed using a special deformation module that uses a spring analogy for the modification of the surface grid nodes.

In general, it is more time consuming to deform the grid. Then, it is preferred to read in the wing geometry and then to remesh within the design procedure using the internal algebraic mesh generator.

\section{FAR-FIELD DRAG TOOL DESCRIPTION}

INTA has employed an in-house tool [4] called DragON to perform the far-field drag analysis. The DragON tool is based on a mixed approach to perform the drag breakdown: the volume approach from van der Vooren and Destarac [5] is applied to extract the profile drag (viscous and shock wave drag components) and a wake plane approach [6] is used to extract the induced or vortex drag.

The viscous and shock wave components of the drag are defined, respectively, by

$$
D_{v}=\int_{\Omega_{v}} \nabla \cdot \vec{f}_{v w} d v ; \quad D_{w}=\int_{\Omega_{w}} \nabla \cdot \vec{f}_{v w} d v
$$

where $\vec{f}_{v w}$ is the irreversible drag vector (see $\left.[4,5]\right) ; \Omega_{v}$ is the volume area of the domain where viscous phenomenon is present (i. e., boundary layers and viscous 


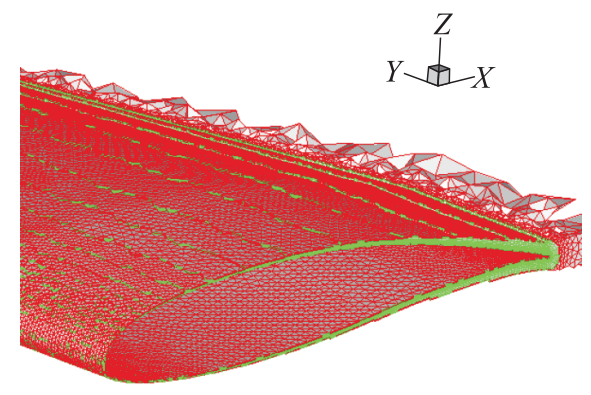

Figure 4 Viscous zone for far-field drag extraction

shear layers or wakes); and $\Omega_{w}$ is the fluid volume which contains shock waves. The region $\Omega_{v}$ is determined by a combination of physical sensors: boundary layers are determined by a cutoff acting on the local norm of the simplified form of the shear stress and shear layers are detected by a sensor which is the function of the turbulent over laminar viscosity ratio. An example of the viscous areas for the here analyzed wing is shown in Fig. 4.

The induced drag is defined in the van der Vooren formulation as

$$
D_{i}(V)=\int_{\Omega_{w}+\Omega_{v}} \nabla \cdot \vec{f}_{v w} d v-\int_{\partial \Omega_{B}}\left(\vec{f}_{i} \cdot \vec{n}\right) d S .
$$

Expressions for the drag vectors $\vec{f}_{i}$ and $\vec{f}_{v w}$ can be found in [5]. This definition ensures the exact balance between the near- and far-field approach for drag extraction. It can be shown that it is possible to improve the prediction of the induced drag given by the above expression by adding a correction term which takes into account the drag produced by numerical sources outside the viscous and shock wave areas. Nevertheless, the determination of the spurious drag zones is somewhat arbitrary and an alternative formulation for the induced drag is employed in DragON.

The approach to extract the induced drag is based on the Maskell integral [6] which has the following expression:

$$
D_{i}(M)=\frac{1}{2} \rho_{\infty} \int_{S_{D}} \psi \xi d S
$$

where $\xi$ is the streamwise vorticity and $\psi$ is the stream function which satisfies a two-dimensional (2D) Poisson equation in a transversal wake plane $S_{D}$. In the $D$ rag $O N$ tool, the stream function is determined by a Green function approach. The wake plane on which the Maskell integral is computed is usually located close to the wing trailing edge in order to avoid as much as possible the vortex 


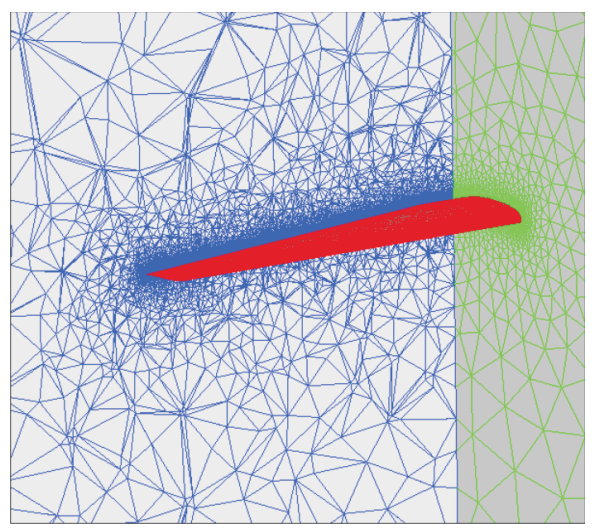

Figure 5 Wake plane SD (blue color) for the Maskell integral

decay produced by numerical dissipation. An example of a cut wake plane for the here analyzed wing is shown in Fig. 5.

\section{PROTOTYPE AIRCRAFT}

The purpose of the study done under INTA-AI-M cooperation was to test a methodology to optimize a military transport aircraft type wing at single flight conditions. There were some trials using multipoint design; but the lack of time avoided a systematic study. The idea was to use the optimization tool CIRCE for a refinement of the initial wing designed with classical engineering methods. Several constraints like the wing surface area - which is fixed - are given and bounded the feasible design space.

The design point is at cruise condition and is given by the following data:

- Mach $=0.45$;

$-h=21,000 \mathrm{ft} ;$ and

$-T=\mathrm{ISA}+0{ }^{\circ} \mathrm{C}$.

The goal is to maximize the aerodynamic efficiency $(L / D)$.

The initial constraints given by $A I-M$ were:

- fixed wing surface;

- maximum wing span: initial wing span;

- minimum thickness-to-chord ratio: $10 \%$; 
- minimum leading edge sweep angle: $9^{\circ}$;

- maximum leading edge angle: $15^{\circ}$;

- minimum taper ratio: 0.2 ;

- maximum taper ratio: 0.5 ; and

- minimum twist: $-10^{\circ}$.

It was not possible to increase the wing span. And the wing surface was fixed. Then, the initial aspect ratio was the maximum possible. It was then difficult to improve the induced drag if the aspect ratio could not be increased. The original wing had a good Oswald factor. The studies of taper ratio performed previously to the optimization procedure showed that the taper ratio could be increased slightly combined with a reduction of leading edge sweep angle. But this improvement was small. Then, it was decided to start the design with studies fixing the plan form to the initial one and leaving free the wing sections geometry and the twist.

\subsection{Optimization Procedure}

The original wing was formed by only one wing section. The optimization was then afforded systematically, starting with a $2 \mathrm{D}$ optimization of this initial wing section. After some tests, it was decided to perform a full three-dimensional (3D) optimization. Due to the number of design variables could be very large and the procedure is a time consuming task, it was decided to start 3D studies using only two airfoils for the wing sections definition. One would define the root and inner part of the wing sections; the other wing section would be the wing tip section.

A scheme of the design by wing section modifications is given in Fig. 6. For a typical wing, no more than 3-4 airfoils are used for the definition of the wing sections. But with an optimization procedure as implemented in CIRCE code, it is possible to provide a very general procedure of wing sections deformation. But the more wing sections are modified, the larger the number of design variables. For this study, only two airfoils were used, as indicated above.

Usually, in one design loop, the wing was only improved slightly compared to the initial one once the cost function achieved a stationary value. Then, this new wing was used as initial wing again. And the design procedure started again, using different cost functions if necessary, and modifying the constraints. A scheme of this methodology is given in Fig. 7.

A typical optimization problem afforded in these test cases was the following:

- minimize $f_{\text {obj }}=100 C_{D}$;

- single point design, Mach $=0.45, \alpha=2^{\circ}$, viscous flow; 


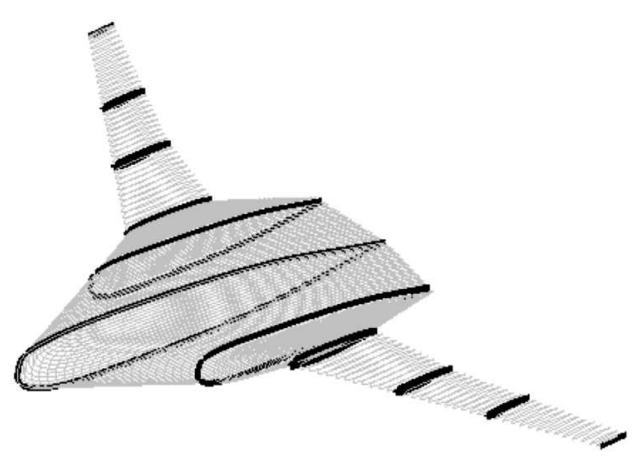

Figure 6 Design by wing sections modifications

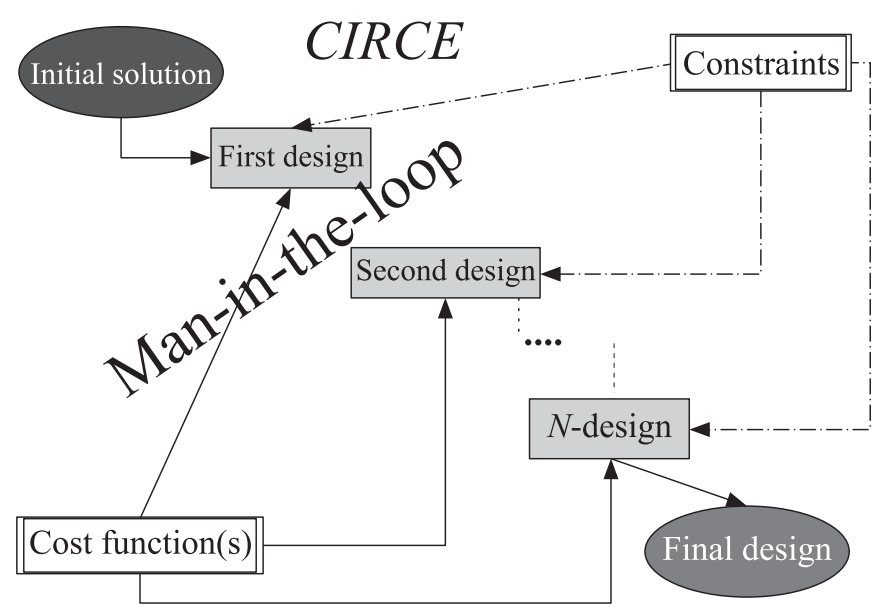

Figure 7 Design procedure

- constraints:

- $\left(\frac{t}{c}\right)_{1} \leq\left(\frac{t}{c}\right)_{\max } \leq\left(\frac{t}{c}\right)_{2}$ at root section;

- $\left(\frac{t}{c}\right)_{3} \leq\left(\frac{t}{c}\right)_{\max } \leq\left(\frac{t}{c}\right)_{4}$ at tip section; and

- $C_{L} \geq 0.650$; 
- design variables:

- 28 design variables: 8 design variables for thickness perturbation functions and 6 design variables for mean line perturbation variables in root section and tip section;

- wing sections: In the central box of the wing the airfoils are similar to the root section. In the outer part of the wing, each section is a linear interpolation of root and tip sections;

- 1 design variable $(\alpha)$ : angle of attack is free;

- gradients: central differences for the computation of gradients.

The constraints in thickness-to-chord ratio are different for both the root and tip sections. It was intended to obtain a wing with a thicker root section than the tip as indicated by AI-M.

In order to reduce the computing time, the grid used for flow analysis was the coarsest possible maintaining a good accuracy in the drag coefficient calculation. A single structured mesh of $320 \times 60 \times 64$ grid cells was used. At the design conditions, the flow was attached. Wall function was used for wall shear forces computation. The results showed the values of $0.6 \leq y^{+} \leq 30$ in the wing surface.

Up to 30 optimization cases were done. The first 4 cases were $2 \mathrm{D}$ optimizations. The best cases obtained were the named 'design17' and 'design30' wings. For the first one, it was applied an optimization in the conditions shown above. The 'design30' wing was a case that used 'design17' as the initial wing and the wing sections were obtained using cubic $B$-splines for the determination of the airfoils.

In most cases, the optimization led to a reduction of the thickness of the initial geometry in order to reduce the viscous drag. Also, there were differences in mean lines. The aerodynamic efficiencies - relative to a reference value of the initial wing (called 'lambda 0.36 ') and some other wings calculated are shown in Fig. 8. The lift coefficients for the maximum values for all wings do not vary so much compared to the initial one. For the 'design8' wing, the root section was slightly thinner than the tip section (12.5\% compared to $12.8 \%$ ). Additionally, the thickness in the trailing edge was so small. The 'design15' wing was thicker at root. The root section had a thickness-to-chord ratio of $13.5 \%$ and the tip section of $12 \%$. Moreover, the pitching moment was slightly more positive than the initial one $(+1.3 \%)$, while for the 'design 8 ' was more negative $(-4.9 \%$ increment at design lift). It was then preferred the 'design 15 ' than 'design8' as the efficiencies of both wings were very similar. Finally, the 'design17' wing was the thinner one and had the largest efficiency. The root section had a thickness-to-chord ratio of $12 \%$ while the wing tip thickness-tochord ratio was only $10 \%$. 


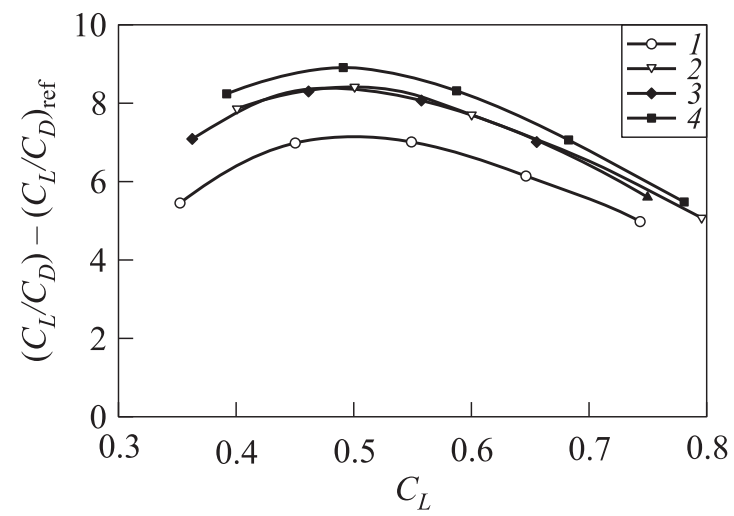

Figure 8 Aerodynamic efficiencies of several wings using CIRCE code for flow computations and a coarse mesh: 1 - lambda $0.36 ; 2$ - lambda 0.36 , design $8,3 \mathrm{D} ; 3-$ lambda 0.36 , design 15, 3D; and 4 - lambda 0.36, design17, 3D

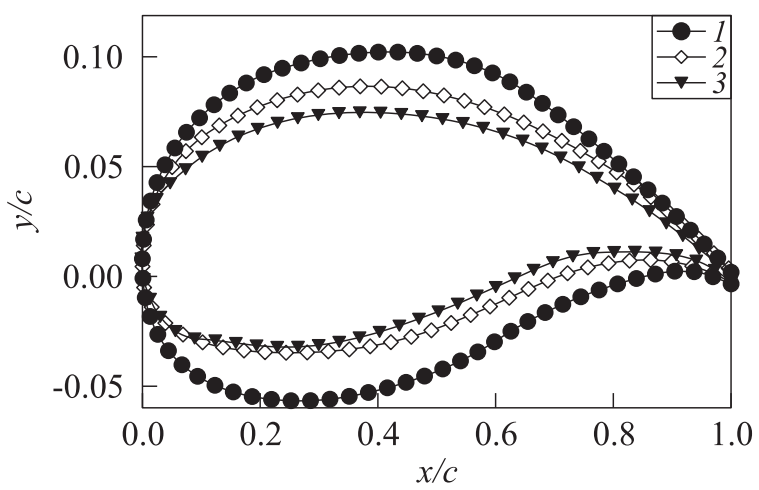

Figure 9 Initial wing section and designed wing sections of 'design17' configuration: 1 - initial wing section; 2 - design 17 , root section; and 3 - design17, tip section

The chosen wing for comparison of the performances at cruise condition was originally the 'design17' wing. Figure 9 shows the differences between the initial wing section and the root and tip sections of the 'design17' wing. The root section of the new wing had a thickness-to-chord ratio of $12 \%$ while the initial wing section was of $16 \%$. The tip wing section had a thickness-to-chord ratio of $10 \%$, i. e., the minimum value imposed by the constraints.

After the design procedure was finished, a new wing — called 'design30' wing — was designed starting with the 'design17' wing and using in this case cubic $B$-splines for the definition of the wing sections. 


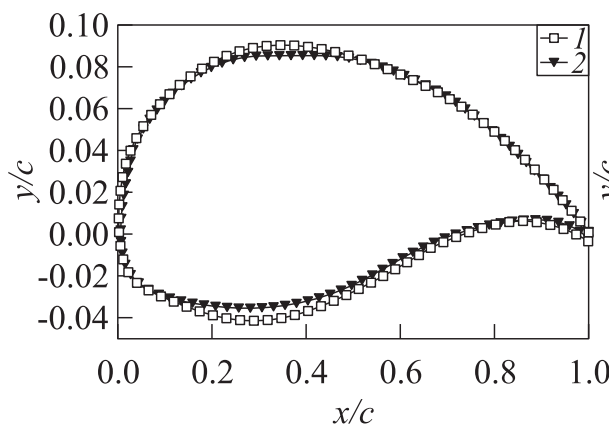

(a)

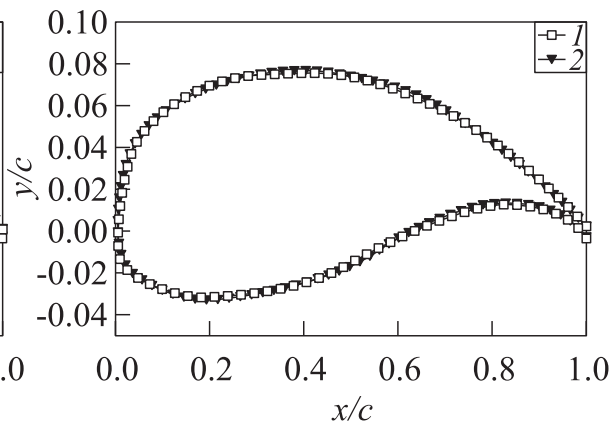

(b)

Figure 10 Designed wing sections of 'design17' (1) configuration compared to 'design30' (2) wing sections: $(a) z=0.0$; and $(b) z=1.0$

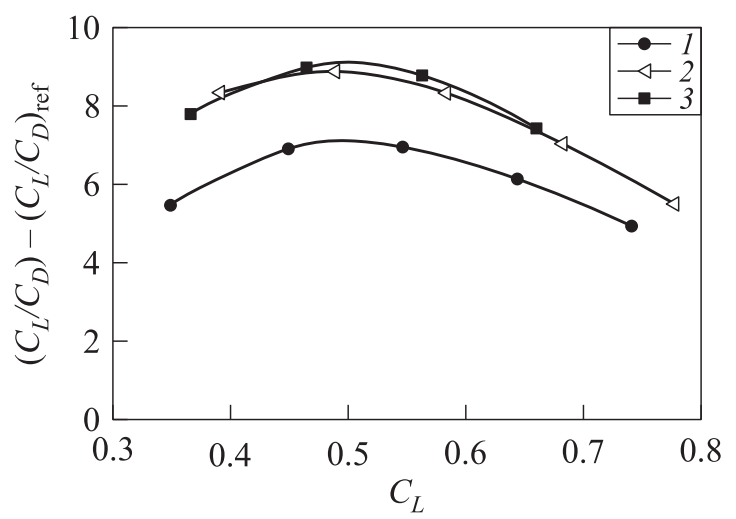

Figure 11 Aerodynamic efficiencies of initial and two designed wings using CIRCE code for flow computations and a coarse mesh: 1 - lambda 0.36; 2 - lambda 0.36, design17, 3D; and 3 - lambda 0.36, design30, 3D

In this case, a multipoint design was done. The cost function chosen was $f_{\text {obj }}=1000\left(\omega_{1} C_{D 1}+\omega_{2} C_{D 2}\right)$ being $\omega_{1}=0.20$ and $\omega_{2}=0.80$. The two points correspond to two lift conditions at the same altitude. Constraints in lift, pitching moment, and thickness-to-chord ratio were imposed. The wing sections are shown in Fig. 10. It can be seen that the root section of 'design30' wing increased its thickness-to-chord ratio (to $13 \%$ ) while the tip section suffered a very small change - except in the lower side trailing edge area - compared to the initial tip section of 'design17' configuration.

A comparison of the aerodynamic efficiencies of these wings - compared to the initial one - is given in Fig. 11. The efficiencies are similar, but in this case, 
the pitching moment variation is smaller for 'design30' wing. The root section is thicker. Then, it would be preferable the 'design30' compared to 'design17.' But a fine analysis of the configuration was only done for 'design17' wing. Then, only the result of this analysis is given in the next section.

\subsection{Validation: Analysis of Original and Designed Wings}

The 'design17' wing was analyzed at the same flow and numerical conditions, then the original wing and both wings were compared.

A validation performed with the same flow solver of $C I R C E$ code, but with a finer mesh than that used for the optimization $(320 \times 96 \times 92$ grid cells and no wall function for wall shear calculation), gave the following results (Table 3 ) at cruise conditions.

In general, the new wing is more efficient at all the lift conditions studied as Fig. 12 shows. Again, the efficiencies are relative to a reference value. The lift coefficients for maximum efficiencies are close to 0.5 in both curves.

The results show that the new wing reduced the drag coefficient up to 12 d.c (drag counts).

The drag coefficient was obtained by pressure integration in the following manner:

$$
\vec{F} \equiv \vec{F}\left(F_{x}, F_{y}, F_{z}\right)=\left(\iint_{S}-\left(p-p_{\infty}\right) \cdot \vec{n} d S+\iint_{S} \overline{\bar{\tau}} \cdot \vec{n} d S\right) .
$$

For obtaining the lift and drag forces, the vector $\vec{F}$ is projected into wind axes. For a symmetric case (no sideslip angle), the force is given, therefore, in the form: $\vec{F} \equiv \vec{F}\left(D, L, F_{z}\right)$ where $D=F_{x} \cos \alpha+F_{y} \sin \alpha$ and $L=-F_{x} \sin \alpha+F_{y} \cos \alpha$, $\alpha$ being the angle of attack.

In order to have more confident results, an analysis of both the original and new designed wing was carried out using the $T A U$ flow solver.

It is important to remark that for the optimization procedure, the geometry of the wing tips of both the original and designed wings was simplified for the meshing procedure employed by CIRCE.

Table 3 Comparison of global coefficients at cruise conditions $\left(C_{L}\right.$ $\left.=C_{L_{\text {cruise }}}\right)$ using $C I R C E$ code results

\begin{tabular}{lcccc}
\hline Geometry & $\Delta \alpha$ & $\Delta C_{M}$ & $\Delta C_{D}$ & $\Delta\left(C_{L} / C_{D}\right)$ \\
\hline Initial & - & - & - & - \\
Design17 & $-0.386^{\circ}$ & -0.0095 & -0.0012 & 1.5905 \\
\hline
\end{tabular}




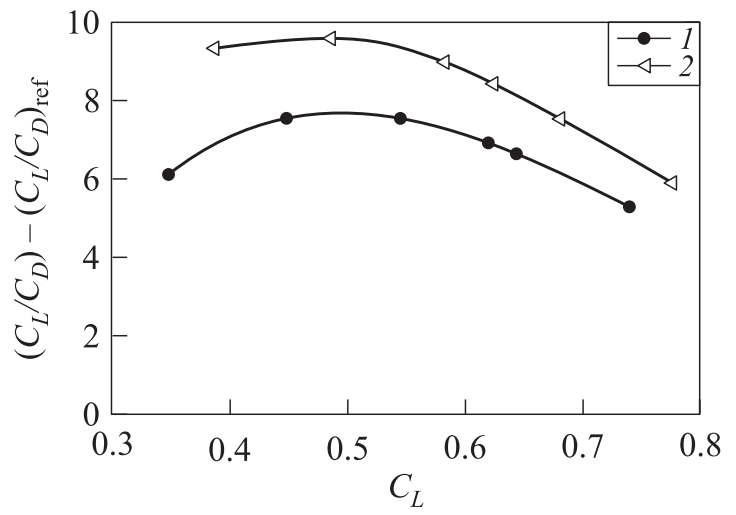

Figure 12 Aerodynamic efficiency curves of the original and 'design17' wings obtained with CIRCE flow solver and fine mesh: 1 - lambda 0.36; and 2 - lambda 0.36 , design $17,3 \mathrm{D}$

When performing the comparison with $T A U$ flow solver, the original wing was analyzed, and the 'design17' wing was modified in the wing tip in order to have a rounded wing tip. This can affect to the induced drag primarily. Additionally, for $T A U$ computations, a one-equation turbulence model (Spalart-Allmaras) was used instead of the algebraic model used by CIRCE; nevertheless, the flow conditions were attached flow.

$T A U$ code computes the forces using the near-field approach in a similar manner than CIRCE code, as explained above. But the DragON tool has also been implemented such that the flow field given by the solver is used to compute the drag force by the far-field method explained in section 3 . The solution of $C I R C E$ code was also used for computing the drag; but here, the results of $T A U$ code will only be shown.

The solution at cruise condition $\left(C_{L}=C_{L_{\text {cruise }}}\right)$ is given in Table 4 .

Comparing the solution given by $T A U$ using the near-field approach with the solution given by $C I R C E$ code, the drag has been reduced to half of the

Table 4 Comparison of drag coefficients using $T A U$ code calculations at cruise conditions $\left(C_{L}\right.$ $\left.=C_{L_{\text {cruise }}}\right)$

\begin{tabular}{lcc}
\hline \multirow{2}{*}{ Geometry } & \multicolumn{2}{c}{$\Delta C_{D}$} \\
\cline { 2 - 3 } & Near-field & Far-field \\
\hline Original & - & - \\
Design17 & -0.00048 & -0.00083 \\
\hline
\end{tabular}


predicted value by $C I R C E: 4.8$ d.c for $T A U$ and 11.7 d.c for CIRCE. It is worth noting that the wings compared were different in the tip region, and this can affect to these calculations.

When using the far-field method, the new wing has a more favorable comparison with the original one: there is a reduction of 8.3 d.c. Unfortunately, there are no results for far-field computations of drag using the CIRCE code results.

It is possible to confirm either by the fine analysis of $C I R C E$ code or $T A U$ code or by using the $\operatorname{DragON}$ tool that the new wing - called 'design17' wing produces less drag at the cruise condition. The polar curves obtained with $C I R C E$ predict an improvement at other lift conditions. At least, it is possible a reduction of 5 d.c at cruise. The far-field calculation increases this value up to 8 d.c.

\section{CONCLUDING REMARKS}

Conclusions about the design procedure are the following:

- a design taking into account viscous flow effects has been shown to be a complex procedure. But it is important to deal with viscous effects in such type of subsonic flow with many constraints for the reduction of drag due to there is no wave drag and the maximum wing aspect ratio is limited to the initial value;

- the improvements of the initial configuration are small in one optimization cycle if all the constraints are to be fulfilled;

- it is necessary to perform several optimization cycles with different initial wings and initial design points. The resultant feasible geometries must then be analyzed at different flow conditions and then their aerodynamic efficiencies can be properly compared to quantify the relative improvement respect to the original wing;

- the design coupling the plan form effects and wing section effects has not been done. It was difficult to obtain wing plan forms different to the initial one without violating the wing area constraint. In terms of leading edge sweep angle or twist distribution, this is a matter of study once a geometry with different wing sections has been chosen;

- the procedure is complex and a man-in-the-loop process. Then, the choice of a new wing configuration requires a large period of time; and

- a design procedure based on an optimization method is likely more efficient - leading to larger reductions of drag — for preliminary design 
studies, with more free parameters for the design. The design shown in this paper was really a refinement of an initial optimum wing. Then, the possibilities of improvement were strongly limited by the constraints posed in the initial wing. Nevertheless, the use of the design procedure based on this optimization code has helped to refine the initial design without violating any of the constraints.

Some conclusions of the fine analysis are the following:

- it was checked that an improvement of the aerodynamic efficiency is possible using this optimization method. Nevertheless, this fine analysis of both the original and the final configuration showed a reduction of the expected improvement. The reason was likely the simplification of the wing geometry in order to perform the design, as well the analysis with other flow solvers or methods captured with larger accuracy several sources of drag as the wing tip vortex - not fully captured within the partial analyses in the design loop; and

- the fine analysis showed almost no reduction in induced drag. The reason was that the initial wing loading was close to the optimum elliptical loading. As the span and the aspect ratio of the wing were constrained to the initial values, it was very difficult to improve this wing loading. The final wing loading was very similar to the initial one. Then, the possibilities of drag reduction were limited to a reduction of drag due to viscous effects.

\section{ACKNOWLEDGMENTS}

This work has been done under contract ME-T-NT-100016 with AIRBUSMilitary. The TAU code is the property of the Deutsches Zentrum für Luft und Raumfahrt (DLR), developed at the Aerodynamics and Flow Technology at Göttingen and Braunschweig, and has been licensed to INTA through a research and development cooperation agreement.

The authors express their gratitude to José Manuel Olalla Sanchez of the Aerodynamics Department, who had also participated in the project doing the procedures concerning the mesh generation for the different geometries.

\section{REFERENCES}

1. Jiménez-Varona, J. 2005. Design of blended wing body configurations using a constrained numerical optimization method. CEAS/KATnet Conference on Key Aerodynamic Technologies. Bremen, Germany. 
2. Vanderplaats, G. N., and F. Moses. 1973. Structural optimization by methods of feasible directions. Comput. Struct. 3:739-755.

3. Vanderplaats, G. N. 1978. CONMIN-a Fortran Program for constrained function minimization. User's Technical Manual. NASA. Technical Report TM X-62,282.

4. Ponsin, J. 2009. Development of a far-field drag extraction tool: DragON. $\mathrm{AT} / \mathrm{TNO} / 4410 / 002 / \mathrm{INTA} / 09$.

5. Destarac, D., and J. van der Vooren. 2004. Drag/thrust analysis of jet-propelled transonic transport aircraft; definition of physical drag components. Aerosp. Sci. Technol. 8:545-556.

6. Giles, M., and R. M. Cummings. 1999. Wake integration for three-dimensional flow field computations: Theoretical development. J. Aircraft. 36(2):357-365. 